\title{
WEYL THEORY AND EXPLICIT SOLUTIONS OF DIRECT AND INVERSE PROBLEMS FOR DIRAC SYSTEM WITH A RECTANGULAR MATRIX POTENTIAL
}

\author{
B. Fritzsche, B. Kirstein, I. Ya. Roitberg and A. L. Sakhnovich
}

Abstract. A non-classical Weyl theory is developed for Dirac systems with rectangular matrix potentials. The notion of the Weyl function is introduced and the corresponding direct problem is solved. Furthermore, explicit solutions of the direct and inverse problems are obtained for the case of rational Weyl matrix functions.

Mathematics subject classification (2010): 34B20, 34L40, 15A15, 93B15.

Keywords and phrases: Weyl function, Weyl theory, Dirac system, rectangular matrix potential, direct problem, inverse problem, pseudo-exponential potential, explicit solution, rational matrix function, nonexpansive matrix function, realization.

\section{REFERENCES}

[1] M. J. Ablowitz, D. J. Kaup, A. C. Newell, H. Segur, The inverse scattering transform Fourier analysis for nonlinear problems, Stud. Appl. Math. 53 (1974) 249-315.

[2] M. J. Ablowitz, H. Segur, Solitons and the inverse scattering transform, SIAM Stud. Appl. Math. 4, Philadelphia, 1981.

[3] D. Alpay, I. Gohberg, M. A. KaAshoek, L. Lerer, A. Sakhnovich, Krein systems, in: Oper. Theory Adv. Appl. 191 (2009), Birkhäuser, 19-36.

[4] D. Alpay, I. Gohberg, M. A. KaAshoek, L. Lerer, A. SaKhnovich, Krein systems and canonical systems on a finite interval: accelerants with a jump discontinuity at the origin and continuous potentials, Integral Equations Operator Theory 68:1 (2010) 115-150.

[5] D. Z. ARov, H. DYM, Direct and inverse problems for differential systems connected with Dirac systems and related factorization problems, Indiana Univ. Math. J. 54:6 (2005) 1769-1815.

[6] D. Z. Arov, H. DyM, J-contractive matrix valued functions and related topics, Encyclopedia of Mathematics and its Applications 116, Cambridge University Press, Cambridge, 2008.

[7] K. ChADAN, P. C. SABATIER, Inverse problems in quantum scattering theory, Springer, New York, 1989.

[8] J. L. CIEŚLIŃSKI, Algebraic construction of the Darboux matrix revisited, J. Phys. A 42:40 (2009), 404003, $40 \mathrm{pp}$.

[9] S. Clark, F. GeSZTESY, Weyl-Titchmarsh $M$-function asymptotics, local uniqueness results, trace formulas, and Borg-type theorems for Dirac operators, Trans. Amer. Math. Soc. 354 (2002) 34753534.

[10] S. Clark, F. GeszTESy, On Self-adjoint and J-self-adjoint Dirac-type Operators: A Case Study, Contemp. Math. 412 (2006) 103-140.

[11] M. M. Crum, Associated Sturm-Liouville systems, Quart. J. Math. Oxford (2) 6 (1955) 121-127.

[12] P. A. DEIFT, Applications of a commutation formula, Duke Math. J. 45 (1978) 267-310.

[13] L. D. Faddeev, L. A. TaKhtajan, Hamiltonian methods in the theory of solitons, Springer, New York, 1986.

[14] B. Fritzsche, B. Kirstein, I. Ya. Roitberg, A. L. Sakhnovich, Weyl matrix functions and inverse problems for discrete Dirac type self-adjoint system: explicit and general solutions, Operators and Matrices 2 (2008) 201-231. 
[15] B. Fritzsche, B. Kirstein, I. Ya. Roitberg, A. L. Sakhnovich, Recovery of Dirac system from the rectangular Weyl matrix function, Inverse Problems 28 (2012), 015010, 18 pp.

[16] B. Fritzsche, B. KiRstein, AND A. L. SAKhnovich, Completion problems and scattering problems for Dirac type differential equations with singularities, J. Math. Anal. Appl. 317 (2006) 510-525.

[17] B. Fritzsche, B. Kirstein, A. L. SAKhnovich, Semiseparable integral operators and explicit solution of an inverse problem for the skew-self-adjoint Dirac-type system, Integral Equations Operator Theory 66 (2010) 231-251.

[18] B. Fritzsche, B. Kirstein, A. L. Sakhnovich, Weyl Functions of Generalized Dirac Systems: Integral Representation, the Inverse Problem and Discrete Interpolation, J. Anal. Math. to appear.

[19] F. GESZTESY, A complete spectral characterization of the double commutation method, J. Funct. Anal. 117 (1993) 401-446.

[20] F. Gesztesy, H. Holden, Soliton equations and their algebro-geometric solutions, Cambridge Studies in Advanced Mathematics 79, Cambridge University Press, Cambridge, 2003.

[21] F. Gesztesy, W. Schweiger, B. Simon, Commutation methods applied to the mKdV-equation, Trans. Amer. Math. Soc. 324 (1991) 465-525.

[22] F. Gesztesy, G. Teschl, On the double commutation method, Proc. Amer. Math. Soc. 124 (1996) $1831-1840$.

[23] I. Gohberg, M. A. KaAshoek, A. L. Sakhnovich, Bound states for canonical systems on the half and full line: explicit formulas, Integral Equations Operator Theory 40:3 (2001) 268-277.

[24] I. Gohberg, M. A. KAAShoek, A. L. SAKHnOvich, Scattering problems for a canonical system with a pseudo-exponential potential, Asymptotic Analysis 29:1 (2002) 1-38.

[25] C. H. GU, H. HU, Z. ZHou, Darboux transformations in integrable systems, Springer Verlag, 2005.

[26] R. E. Kalman, P. Falb, M. Arbib, Topics in mathematical system theory, McGraw-Hill, New York, 1969.

[27] A. Kostenko, A. Sakhnovich, G. Teschl, Commutation Methods for Schrödinger Operators with Strongly Singular Potentials, Math. Nachr. 285 (2012) $392-410$.

[28] M. G. KREIN, Continuous analogues of propositions on polynomials orthogonal on the unit circle (Russian), Dokl. Akad. Nauk SSSR 105 (1955) 637-640.

[29] M. G. KREIN, On a continual analogue of a Christoffel formula from the theory of orthogonal polynomials (Russian), Dokl. Akad. Nauk SSSR (N.S.) 113 (1957) 970-973.

[30] M. G. KReIn, Topics in differential and integral equations and operator theory, Oper. Theory Adv. Appl. 7, Birkhäuser, Basel-Boston, 1983.

[31] P. Lancaster, L. Rodman, Algebraic Riccati equations, Clarendon Press, Oxford, 1995.

[32] B. M. Levitan, I. S. Sargsjan, Sturm-Liouville and Dirac operators, Mathematics and its Applications 59, Kluwer, Dordrecht, 1990.

[33] V. A. Marchenko, Sturm-Liouville operators and applications, Oper. Theory Adv. Appl. 22, Birkhäuser, Basel, 1986.

[34] V. A. Marchenko, Nonlinear equations and operator algebras, Reidel Publishing Co., Dordrecht, 1988.

[35] V. B. Matveev, M. A. Salle, Darboux transformations and solitons, Springer Verlag, Berlin, 1991.

[36] V. P. Potapov, The multiplicative structure of J-contractive matrix functions, Amer. Math. Soc. Transl. 15 (1960) 131-243.

[37] A. L. SAKHNOVICH, A nonlinear Schrödinger equation on the semiaxis and a related inverse problem, Ukrain. Math. J. 42:3 (1990) 316-323.

[38] A. L. SAKHnovich, The Goursat problem for the sine-Gordon equation and the inverse spectral problem, Russ. Math. Iz. VUZ 36:11 (1992) 42-52.

[39] A. L. Sakhnovich, Dressing procedure for solutions of nonlinear equations and the method of operator identities, Inverse Problems, 10 (1994) 699-710.

[40] A. L. Sakhnovich, Inverse spectral problem related to the $N$-wave equation, in: M. G. Krein volume, Oper. Theory Adv. Appl. 117 (2000), Birkhäuser, Basel, 323-338.

[41] A. L. SAKHNOVICH, Dirac type and canonical systems: spectral and Weyl-Titchmarsh fuctions, direct and inverse problems, Inverse Problems 18 (2002) 331-348.

[42] A. L. SAKhNOvich, Second harmonic generation: Goursat problem on the semi-strip, Weyl functions and explicit solutions, Inverse Problems 21:2 (2005) 703-716. 
[43] A. L. SAKhNovich, On the GBDT version of the Bäcklund-Darboux transformation and its applications to the linear and nonlinear equations and spectral theory, Mathematical Modelling of Natural Phenomena 5:4 (2010) 340-389.

[44] L. A. SakHNovich, On the factorization of the transfer matrix function, Sov. Math. Dokl. 17 (1976) 203-207.

[45] L. A. SAKhnovich, Factorisation problems and operator identities, Russian Math. Surv. 41 (1986) $1-64$.

[46] L. A. S A KhNOvich, Integral equations with difference kernels on finite intervals, Oper. Theory Adv. Appl. 84, Birkhäuser, Basel-Boston-Berlin, 1996.

[47] L. A. SAKHNOVICH, Spectral theory of canonical differential systems. Method of operator identities, Oper. Theory Adv. Appl. 107, Birkhäuser, Basel-Boston, 1999.

[48] L. A. Sakhnovich, Matrix finite-zone Dirac-type equations, J. Funct. Anal. 193:2 (2002), 385-408.

[49] G. TESCHL, Jacobi operators and completely integrable nonlinear lattices, Mathematical Surveys and Monographs, Amer. Math. Soc., Rhode Island, 2000.

[50] G. Teschl, Mathematical Methods in Quantum Mechanics; With Applications to Schrödinger Operators, Graduate Studies in Mathematics, Amer. Math. Soc., Rhode Island, 2009.

[51] V. E. Zakharov, A. V. Mikhailov, On the integrability of classical spinor models in twodimensional space-time, Comm. Math. Phys. 74 (1980) 21-40.

[52] V. E. Zaharov, A. B. Shabat, On soliton interaction in stable media, JETP 64 (1973) 1627-1639. 